\title{
Towards individualized radiation therapy in multiple myeloma
}

\section{Felix Momm, ${ }^{1}$ Christine Greil ${ }^{2}$ and Henning Schäfer ${ }^{3}$}

${ }^{1}$ Department of Radiation Oncology, Ortenau Klinikum Offenburg-Kehl, Teaching Hospital of Albert-Ludwigs University Freiburg, Offenburg; ${ }^{2}$ Department of Hematology, Oncology and Stem Cell Transplantation, Medical Center, Faculty of Medicine, University of Freiburg, Freiburg and ' 3 Department of Radiation Oncology, Medical Center, Faculty of Medicine, University of Freiburg, German Cancer Consortium (DKTK) Partner Site Freiburg, German Cancer Research Center (DKFZ), Heidelberg, Germany

E-mail: FELIX MOMM - felix.momm@ortenau-klinikum.de

doi:10.3324/haematol.2019.243451

$\mathrm{F}$ ortunately, recent progress in systemic treatment has prolonged the median overall survival of myeloma patients. ${ }^{1,2}$ However, this leads to increasing numbers of radiation cycles being administered for supportive reasons (e.g. pain, stability). Although radiation can result in hematopoietic insufficiency in a dose-dependent manner, leading to ineligibility for systemic treatment, the best radiation regimen providing optimal local control and minimized bone marrow toxicity is still under investigation. The current guidelines for radiotherapy in patients diagnosed with multiple myeloma were summarized in a critical review by the International Lymphoma Radiation Oncology Group in 2018. ${ }^{3}$ In their letter published in this issue of Haematologica, Elhammali et al. contribute the large and high-value experience of the MD Andersson Cancer Center to this ongoing discussion. ${ }^{4}$ After analysis of treatment, toxicities and tumor control in 772 myeloma patients with 1,513 irradiated lesions and a median follow-up of 65.6 months the authors claim that radiation doses as low as 20-25 Gy were sufficient to avoid reirradiation in more than $97 \%$ of all cases. However, in univariate analysis, a biologically effective dose assuming an $\alpha / \beta$ ratio of $10 \mathrm{~Gy}\left(\mathrm{BED}_{10}\right)$ of $<28 \mathrm{~Gy}$ was associated with an increased risk of reirradiation. As the authors state themselves, the study is limited by the well-known bias of retrospective data with a long observation period. ${ }^{4}$ From our perspective, the most important problems seem to be the comparatively weak endpoint of reirradiation and the selection bias of how dose was determined in the individual patient. ${ }^{4}$ Our own clinical experience shows that lower doses are preferentially given to elderly or frail patients, in order to save treatment time and reduce acute toxicity, or in cases of small lesions with low tumor burden. In fragile patients reirradiation is often not performed as these patients may die prior to local tumor recurrence because of systemic disease progression or relevant comorbidities and the reirradiation is therefore underestimated. In patients with small lesions, a lower irradiation dose may probably be sufficient to achieve local tumor control. Despite the reported data, we would therefore still recommend higher doses of 30-40 Gy to large lesions as stated by the International Lymphoma Radiation Oncology Group. ${ }^{3}$ Especially when treating critical lesions of the spine or the skull base as well as lesions particularly prone to fractures, it is essential to reach stable local tumor control. In this context we suggest that toxicity should be avoided by shrinking the irradiation field rather than by reducing dose. Targeted dosage to bone lesions or extramedullary tumors will not compromise bone marrow function relevantly.

Nevertheless, the reported routine clinical records of a reference center with a high number of cases ${ }^{4}$ still contribute valid arguments to the ongoing discussion: The authors could show that in this selected cohort comparatively low doses were sufficient for a high rate of tumor control, emphasizing that selected plasmacytoma sites with low tumor burden might definitely be effectively irradiated with doses as low as 20-25 Gy, and that in advanced disease stage, lower doses and therefore shorter time of treatment are still sufficient for local control. ${ }^{4}$

The best approach is probably to apply high doses precisely to sites with large tumor burden and to reduce doses in sites with less or disseminated tumor and if functional bone marrow may be affected. With modern inten-
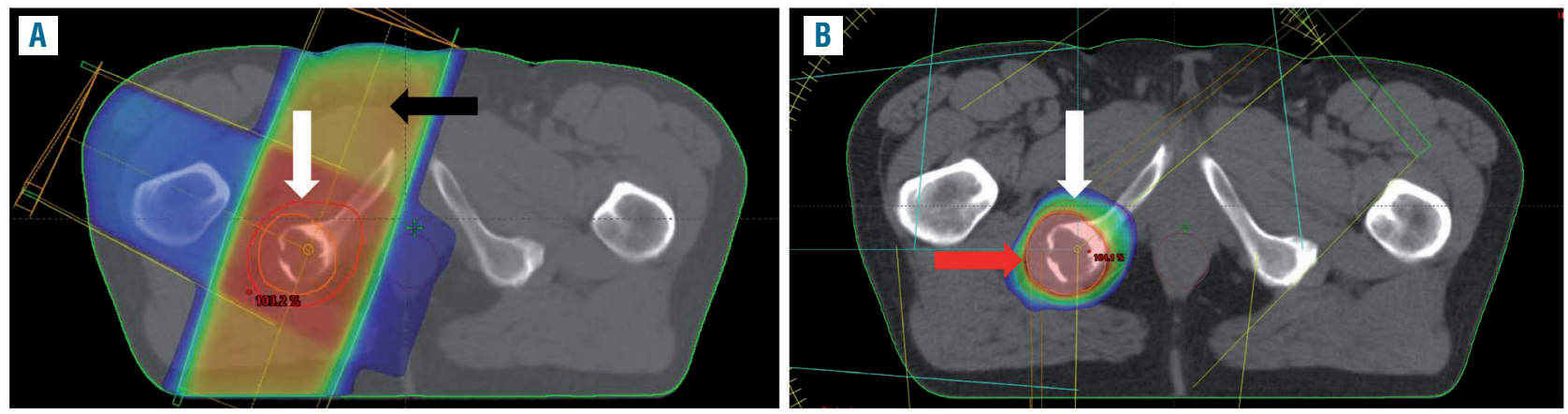

Figure 1. Planning computed tomography scanning of a myeloma lesion in the right ischium. The dose distribution is depicted by a color wash from red (= high dose) to dark blue (= low dose). (A) Conventional technique: 50 Gy in 20 fractions of 2.5 Gy to complete target volume. Black arrow: areas of high dose out of target volume (red line/white arrow). (B) Volume modulated arc therapy (VMAT) technique. Red line/white arrow: main target volume 40 Gy in 20 fractions of 2.0 Gy; orange line/red arrow: integrated boost target volume 50 Gy in 20 fractions of 2.5 Gy. The complete dose of each fraction is delivered simultaneously integrated to both target volumes. 
sity-modulated radiotherapy or volume-modulated arc therapy such dose distributions can be routinely attained (Figure. 1). Considering possibilities of modern imaging, including magnetic resonance imaging and positron emission tomography/computed tomography, which are able to depict myeloma lesions very precisely, highly conformal radiotherapy techniques can be applied with a comparatively low risk of missing the target. ${ }^{5}$ Thus, even highprecision stereotactic radiotherapy or radiosurgery can be considered for the treatment of critical multiple myeloma lesions in the spine. ${ }^{6}$ Of course, using such radiation techniques, effective systemic therapy is an essential part of the interdisciplinary treatment concept.

Radiotherapy is the treatment of choice for multiple myeloma in two situations: as curative treatment for single plasmacytoma lesions and as palliation for local symptoms due to certain bone or extramedullary lesions. In both situations local tumor control is most important and, therefore, the radiation dose sought should not differ. In fact, the decision regarding each patient's or each lesion's dose should also be taken on the basis of that patient's general condition and life expectancy. In this context, specific help for dose decisions can be obtained by scores, such as the one developed by Rades et al. ${ }^{7}$

Furthermore, and probably most importantly, considering multiple myeloma and also solitary plasmacytoma as a systemic disease, radiation doses and volumes should not adversely affect the administration of essential systemic therapies by being toxic to the bone mar- row. To ensure this, patients diagnosed with multiple myeloma should be treated in an interdisciplinary manner by oncologists and radiation oncologists together. In summary, the work by Elhammali et al. may not cause a paradigm shift, but it does contribute important data with regards to a concept of individualized radiation therapy.

\section{References}

1. Soekojo CY, Kumar SK. Stem-cell transplantation in multiple myeloma: how far have we come? Ther Adv Hematol. 2019;10: 2040620719888111.

2. Piechotta V, Jakob T, Langer P, et al. Multiple drug combinations of bortezomib, lenalidomide, and thalidomide for first-line treatment in adults with transplant-ineligible multiple myeloma: a network meta-analysis. Cochrane Database Syst Rev. 2019;2019(11).

3. Tsang RW, Campbell BA, Goda JS, et al. Radiation therapy for solitary plasmacytoma and multiple myeloma: guidelines from the International Lymphoma Radiation Oncology Group. Int J Radiat Oncol Biol Phys. 2018;101(4):794-808.

4. Elhammali A, Amini B, Ludmir EB, et al. New paradigm for radiation in multiple myeloma: lower yet effective dose to avoid radiation toxicity. Haematologica. 2020;105(7):e355-e357.

5. Zhu Q, Zou X, You R, et al. Establishment of an innovative staging system for extramedullary plasmacytoma. BMC Cancer. 2016; 16(1):777.

6. Miller JA, Balagamwala EH, Chao ST, et al. Spine stereotactic radiosurgery for the treatment of multiple myeloma. J Neurosurg Spine. 2017;26(3):282-290.

7. Rades D, Conde-Moreno AJ, Cacicedo J, et al. A predictive tool particularly designed for elderly myeloma patients presenting with spinal cord compression. BMC Cancer. 2016;16:292. 